\title{
Classical Neumann Problems for Hessian equations and Alexandrov-Fenchel's inequalities
}

\author{
Guohuan Qiu * Chao Xia $^{\dagger}$
}

May 11, 2018

\begin{abstract}
Recently, the first named author together with Xinan Ma 11, have proved the existence of the Neumann problems for Hessian equations. In this paper, we proceed further to study classical Neumann problems for Hessian equations. We prove here the existence of classical Neumann problems under the uniformly convex domain in $\mathbb{R}^{n}$. As an application, we use the solution of the classical Neumann problem to give a new proof of a family of Alexandrov-Fenchel inequalities arising from convex geometry. This geometric application is motivated from Reilly [17.
\end{abstract}

\section{Introduction}

Let $\Omega$ be a bounded domain in $\mathbb{R}^{n}$ with smooth boundary $\partial \Omega$. It is well-known that for two sufficiently regular function $f$ on $\bar{\Omega}$ and $\varphi$ on $\partial \Omega$, the following classical Neumann boundary value problem for Poisson's equation:

$$
\begin{cases}\Delta u=f(x) & \text { in } \Omega, \\ u_{\nu}=\varphi(x) & \text { on } \partial \Omega\end{cases}
$$

admits a classical solution $u$, which is unique up to an additive constant, if and only if

$$
\int_{\Omega} f d x=\int_{\partial \Omega} \varphi d \mu
$$

Here $\nu$ is the outward unit normal of $\partial \Omega$ and $u_{\nu}=\frac{\partial u}{\partial \nu}$ is the normal derivative of $\nu$. This result was proved by using the Fredholm alternative from functional analysis, See e.g. [6], pp. 130. See also a recent paper by Nardi [13].

\footnotetext{
*Department of Mathematics, University of Science and Technology of China, Hefei, P. R. China Email: guohuan@ustc.edu.cn. The research of GQ was supported by China Scholarship Council.

${ }^{\dagger}$ School of Mathematical Sciences, Xiamen University, 361005, Xiamen, China Email: chaoxia@xmu.edu.cn. Research of CX is supported in part by the Fundamental Research Funds for the Central Universities (Grant No. 20720150012), NSFC (Grant No. 11501480).
} 
It is natural to ask whether similar result holds for $k$-Hessian equation $\sigma_{k}\left(D^{2} u\right)=f$. The Neumann type problems for Monge-Ampere type equations have been well studied by Lions-Trudinger-Urbas [10]. Trudinger [20] considered and proved the existence for the Neumann type problem for $k$-Hessian equation in the case when the domain is a ball and he conjectured similar result holds for general uniformly convex domains. In a recent paper [11, the fist named author and Ma gave an affirmative answer to Trudinger's conjecture. Particularly, the Neumann type boundary condition in [1] is $u_{\nu}=\varphi(x, u)$, where $\frac{\partial \varphi}{\partial u} \leq-c_{0}<0$ for some positive $c_{0}$.

It is clear that the assumption on $\varphi$ excludes the case that $\varphi(x, u)$ only depends on $x$, i.e., $\varphi(x, u)=\varphi(x)$. In this paper, we will study this case, namely, the classical Neumann boundary value problem for the $k$-Hessian equation:

$$
\begin{cases}\sigma_{k}\left(D^{2} u\right)=f(x) & \text { in } \Omega, \\ u_{\nu}=\varphi(x) & \text { on } \partial \Omega .\end{cases}
$$

It turns out that the existence may not hold for general $f(x)$ and $\varphi(x)$. This is because they should satisfy some compatibility condition as (2). In the case $k=n$, Lions-Trudinger-Urbas [10] showed that for sufficient regular $f$ and $\varphi$ on $\bar{\Omega}$ with $f>0$, there exists a pair $(\lambda, u)$ satisfying

$$
\begin{cases}\operatorname{det}\left(D^{2} u\right)=f(x) & \text { in } \Omega, \\ u_{\nu}=\lambda+\varphi(x) & \text { on } \partial \Omega .\end{cases}
$$

Here $\lambda$ is a unique constant while $u$ is unique up to an additive constant.

Our main result in this paper is the following

Theorem 1. Let $\Omega$ be a $C^{4}$ bounded, uniformly convex domain in $\mathbb{R}^{n}$. Let $f \in C^{2}(\bar{\Omega})$ with $f>0$ and $\varphi \in C^{3}(\bar{\Omega})$. Then there is a unique constant $\lambda$ and a unique $k$ admissible solution $u \in C^{3, \alpha}(\bar{\Omega})$ up to an additive constant, satisfying

$$
\begin{cases}\sigma_{k}\left(D^{2} u\right)=f(x) & \text { in } \Omega, \\ u_{\nu}=\lambda+\varphi(x) & \text { on } \partial \Omega .\end{cases}
$$

A solution $u$ is called $k$-admissible if the eigenvalue of the Hessian matrix $D^{2} u$ belongs to $\Gamma_{k}^{+}$, see Section 2 .

We remark that unlike the case $k=1$, we have no explicit expression for $\lambda$. However, it is easy to see a lower bound for $\lambda$ :

$$
\lambda \operatorname{Area}(\partial \Omega) \geq n \int_{\Omega}\left(\frac{f(x)}{C_{n}^{k}}\right)^{\frac{1}{k}} d x-\int_{\partial \Omega} \varphi(x) d \mu .
$$

Therefore, the classical Neumann problem for $k$-Hessian equations (3) may have no solutions. For example, in the case that $f=1$ and $\varphi=0, \lambda$ has to be a nonzero constant by virtue of (6).

Let us illustrate the idea of the proof of Theorem 1. On one hand, Fredholm alternative is not applicable on (5) as in the classical Neumann problem 
for Poisson's equation (11), for we deal with fully nonlinear partial differential equations. On the other hand, it is impossible to get a uniform bound for the solutions to (5) since a solution plus any constant is still a solution. Thus we can not use continuity method to get the existence. In order to overcome this difficulty we use a perturbation argument. We first consider for any $\epsilon>0$ the following boundary value problem

$$
\begin{cases}\sigma_{k}\left(D^{2} u\right)=f(x) & \text { in } \Omega \\ u_{\nu}=-\epsilon u+\varphi(x) & \text { on } \partial \Omega .\end{cases}
$$

The result in [11] gives us the existence of $u_{\epsilon}$ for (7). We then prove a gradient estimate for $u_{\epsilon}$ independent of $\epsilon$. Once we get this, we shall have all the regularity estimate and by letting $\epsilon \rightarrow 0$ we obtain a solution of (5). This kind of argument has been used in [10] when $k=n$. Their a priori gradient estimate heavily depends on the convexity of the solutions. However, in general $k<n$ case we have no convexity. Instead we use directly maximum principle on some good choice of test functions. The choice of test functions is motivated from [12, see also [11.

A motivation to study such classical Neumann problem for $k$-Hessian equations is to prove the Alexandrov-Fenchel inequalities [1] which are of fundamental importance in the theory of convex geometry. For an introduction of Alexandrov-Fenchel inequalities in convex geometry we refer to Schneider's encyclopedia book [18]. In the past several decades, many mathematicians study such inequalities from the viewpoint of PDEs. Particularly, Trudinger [21] tried to use the Dirichlet problems for $k$-Hessian equations and $k$-curvature equations to reach the following special cases of Alexandrov-Fenchel inequalities between two quermassintegrals for a bounded (possibly non-convex) domain $\Omega \subset \mathbb{R}^{n}$ :

$$
\left(\frac{V_{n-1-k}(\Omega)}{V_{n-1-k}(B)}\right)^{\frac{1}{n-1-k}} \geq\left(\frac{V_{n-1-l}(\Omega)}{V_{n-1-l}(B)}\right)^{\frac{1}{n-1-l}}, \quad n-1 \geq k>l \geq-1,
$$

where $B$ is the unit ball in $\mathbb{R}^{n}$, and $V_{n-1-k}(\Omega)$ is the $k$-th quermassintegrals of $\Omega$. Guan and Li [8] used inverse mean curvature type flow (parabolic PDEs) to prove inequalities (8) for star-shaped domains. Inspired by Gromov's proof [7] of the isoperimetric inequality, Chang and Wang 4] established inequalities (8) for $l=-1$ and $k=1,2$ when $\Omega$ is $(k+1)$-convex by using a solution of some PDE from the optimal transport, see also [14 for any $k$. They 3 also proved (8) for general $k$ for $(k+1)$-convex domains with non-optimal constants. Cabré [2] used the Neummann problem for Possion's equation (11) with $f=1$ and $\varphi=$ constant plus the Alexandrov-Bakelman-Pucci (ABP) estimate to give a very simple proof of the classical isoperimetric inequality in the Euclidean space.

The above results have a common feature that two geometric quantities are compared. From the theory of convex geometry, we know that the AlexandrovFenchel inequalities can link three quermassintegrals. By applying Reilly's formula [15, 16] on the solution of the classical Neumann problem for Possion's 
equation (11) with $f=1$ and $\varphi=$ constant, Reilly [17] gave a new proof of the following Minkowski's inequality for convex domains in the Euclidean space,

$$
\operatorname{Area}(\partial \Omega)^{2} \geq \frac{n}{n-1} \operatorname{Vol}(\Omega) \int_{\partial \Omega} H d \mu,
$$

where $H$ is the mean curvature of $\partial \Omega$. A similar result has been proved recently by the second author [23] in the hyperbolic space.

In the same spirit, we can apply Reilly's high order formula on the solution of the Neumann problem for the $k$-Hessian equation (5) with $f=1$ and $\varphi=0$ to give a new proof of the following special Alexandrov-Fenchel's inequalities for convex domains in $\mathbb{R}^{n}$.

Theorem 2. Let $\Omega$ be a smooth bounded uniformly convex domain in $\mathbb{R}^{n}$. For any $1 \leq k \leq n-1$, denote by $\sigma_{k}(h)$ the $k$-th mean curvature of $\partial \Omega$. Then

$$
\operatorname{Area}(\partial \Omega)^{k+1} \geq \frac{n^{k}}{C_{n-1}^{k}} \operatorname{Vol}(\Omega)^{k} \int_{\partial \Omega} \sigma_{k}(h) d \mu .
$$

Equality holds if and only if $\Omega$ is a ball in $\mathbb{R}^{n}$.

In the case $k=n-1$, since $\int_{\partial \Omega} \sigma_{n-1}(h) d \mu$ is a dimensional constant, we get the isoperimetric inequality for convex domains in $\mathbb{R}^{n}$.

We remark that recently Wang-Zhang [22] and Xia-Zhang [24] gave new proofs of several geometric inequalities via the ABP method for convex domains in $\mathbb{R}^{n}$.

\section{Preliminaries}

In this section, we review fundamental concepts and properties for the $k$-Hessian operators. For the proof of the facts below, we refer to Garding [5], Reilly [15] or Guan 9 .

The $k$-th elementary symmetric function for $\lambda=\left(\lambda_{1}, \ldots, \lambda_{n}\right) \in \mathbb{R}^{n}$ is defined as

$$
\sigma_{k}(\lambda):=\sum_{i_{1}<i_{2}<\cdots<i_{k}} \lambda_{i_{1}} \lambda_{i_{2}} \cdots \lambda_{i_{k}}, \quad 1 \leq k \leq n .
$$

Let $\mathcal{S}_{n}$ be the set of all symmetric $n \times n$ matrices. The $k$-th elementary symmetric function for $A \in \mathcal{S}_{n}$ is

$$
\sigma_{k}(A):=\sigma_{k}(\lambda(A)), \quad \lambda(A) \text { is the eigenvalue of } A .
$$

The Garding cone $\Gamma_{k}^{+}$is defined as

$$
\Gamma_{k}^{+}=\left\{\lambda \in \mathbb{R}^{n} \mid \sigma_{i}(\lambda)>0, \text { for } 1 \leq i \leq k\right\} .
$$

We say $A \in \mathcal{S}_{n}$ belongs to $\Gamma_{k}^{+}$if its eigenvalue $\lambda(A) \in \Gamma_{k}^{+}$. We use the convention that $\sigma_{0}=1$. 
For $A \in \mathcal{S}_{n}, \sigma_{k}(A)$ can be expressed as

$$
\sigma_{k}(A)=\frac{1}{k !} \sum_{\substack{i_{1}, \cdots i_{k} \\ j_{1}, \cdots, j_{k}}} \delta_{j_{1} \cdots j_{k}}^{i_{1} \cdots i_{k}} A_{i_{1} j_{1}} \cdots A_{i_{k} j_{k}}
$$

where $\delta_{j_{1} \ldots j_{k}}^{i_{1} \ldots i_{k}}$ is the generalized Kronecker symbol. The $k$-th Newton transformation for $A \in \mathcal{S}_{n}$ is the matrix

$$
\left[T_{k}\right]_{i j}(A):=\frac{\partial \sigma_{k+1}(A)}{\partial A_{i j}} .
$$

It is well-known that for $A \in \Gamma_{k}^{+}$, the following Newton-Maclaurin inequalities hold:

$$
\left(\frac{\sigma_{k}(A)}{C_{n}^{k}}\right)^{\frac{1}{k}} \geq\left(\frac{\sigma_{l}(A)}{C_{n}^{l}}\right)^{\frac{1}{l}}, \quad 1 \leq k<l \leq n
$$

Definition 3. For $A_{1}, A_{2}, \cdots A_{k} \in \mathcal{S}_{n}$, the polarization of $\sigma_{k}$ is defined to be

$$
\sigma_{k}\left(A_{1}, \cdots, A_{k}\right):=\frac{1}{k !} \sum_{\substack{i_{1}, \cdots i_{k}, j_{1}, \cdots, j_{k}}} \delta_{j_{1} \cdots j_{k}}^{i_{1} \cdots i_{k}}\left(A_{1}\right)_{i_{1} j_{1}} \cdots\left(A_{k}\right)_{i_{k} j_{k}} .
$$

The mixed $k$-th Newton transformation is defined as

$$
\left[T_{k}\right]_{i j}\left(A_{1}, \cdots, A_{k}\right):=\frac{1}{k !} \sum_{\substack{i_{1}, \cdots i_{k}, j_{1}, \cdots, j_{k}}} \delta_{j j_{1} \cdots j_{k}}^{i i_{1} \cdots i_{k}}\left(A_{1}\right)_{i_{1} j_{1}} \cdots\left(A_{k}\right)_{i_{k} j_{k}} .
$$

It is clear by definition that $\sigma_{k}\left(A_{1}, \cdots, A_{k}\right)$ and $\left[T_{k}\right]_{i j}\left(A_{1}, \cdots, A_{k}\right)$ is multilinear with respect to each variables. Also we have

$$
\sigma_{k}(A)=\sigma_{k}(A, \cdots, A), \quad\left[T_{k}\right]_{i j}(A)=\left[T_{k}\right]_{i j}(A, \cdots, A),
$$

and

$$
(k+1) \sigma_{k+1}(A)=\sum_{i, j} A_{i j}\left[T_{k}\right]_{i j}(A) .
$$

From the multilinear property, we see that for two matrices $A_{1}, A_{2} \in \mathcal{S}_{n}$,

$$
\sigma_{k}\left(A_{1}+A_{2}\right)=\sum_{l=0}^{k} C_{k}^{l} \sigma_{l}(\overbrace{A_{1}, \cdots, A_{1}}^{l}, A_{2}, \cdots, A_{2})
$$

and

$$
\left[T_{k}\right]_{i j}\left(A_{1}+A_{2}\right)=\sum_{l=0}^{k} C_{k}^{l}\left[T_{l}\right]_{i j}(\overbrace{A_{1}, \cdots, A_{1}}^{l}, A_{2}, \cdots, A_{2}) .
$$

For a $C^{2}$ function $u$ on $\mathbb{R}^{n}$, we have the following 


\section{Proposition 4.}

(i) If $\lambda\left(D^{2} u\right) \in \Gamma_{k}^{+}$, then $\left(\frac{\partial \sigma_{k}}{\partial u_{i j}}\left(D^{2} u\right)\right)$ is positive definite, and $\sigma_{k}^{\frac{1}{k}}\left(D^{2} u\right)$ is concave with respect to $D^{2} u$.

(ii) $T_{k}\left(D^{2} u\right)$ is divergence free, i.e.,

$$
\sum_{j} \partial_{j}\left(\left[T_{k}\right]_{i j}\right)\left(D^{2} u\right)=0
$$

\section{Classical Neumann problems}

In this section we study (5) and prove Theorem 1 is clear that if $u$ is a solution then $u+c$ is also a solution of (55). Hence we can not expect a $C^{0}$ estimate for $u$. We note that the $C^{1}$ and $C^{2}$ estimates proved in [11, is still true for (5). However these estimates depend on the $C^{0}$ estimate. The main task here is to find a gradient estimate which does not depend on the $C^{0}$ estimate. As described in the introduction, we will consider the perturbed Neumann problem (77) and establish the following gradient estimate.

Proposition 5. Let $\Omega$ be a smooth bounded, uniformly convex domain in $\mathbb{R}^{n}$. Let $f \in C^{2}(\bar{\Omega})$ with $f>0$ and $\varphi \in C^{3}(\bar{\Omega})$. Let $\epsilon>0$ be any positive constant. Then the Neumann problem (7) admits a unique $k$ addmissible solution $u_{\epsilon}$. Moreover,for sufficiant small constants $\epsilon>0$, there exists a constant $C$, depending on $k, n,\|f\|_{C^{1}},\|\varphi\|_{C^{3}}$, and the uniform convexity of $\partial \Omega$, but independent of $\epsilon$ and $\left\|u_{\epsilon}\right\|_{C^{0}}$, such that

$$
\begin{gathered}
\sup _{\bar{\Omega}}\left|\nabla u_{\epsilon}\right| \leq C, \\
\sup _{\bar{\Omega}}\left|u_{\epsilon}-f_{\Omega} u_{\epsilon}\right| \leq C .
\end{gathered}
$$

Here $f_{\Omega} u_{\epsilon}=\frac{1}{\operatorname{Vol}(\Omega)} \int_{\Omega} u_{\epsilon}$.

We give two remarks before the proof.

Remark 6 .

(i) Once we have Proposition 5 it is standard to give a Schauder type estimate independent of $\epsilon$ :

$$
\left\|u_{\epsilon}-f_{\Omega} u_{\epsilon}\right\|_{C^{2, \alpha}} \leq C .
$$

(ii) This kind of gradient estimate relies heavily on the special structure of (7) and the uniform convexity of $\partial \Omega$. For general case, a $C^{0}$ estimate of $u$ is indispensable to get a gradient estimate. 
In the following we will use the notation $F=\sigma_{k}, F^{i j}=\frac{\partial \sigma_{k}}{\partial u_{i j}}$ and we sum over the repeated indices.

Proof of Proposition 5. The existence part has been proved in [11, Theorem 1.1. We now prove the a priori estimate independent of $\epsilon$. For simplicity, we omit the subscription for $u_{\epsilon}$.

Step 1 . We prove by maximum principle

$$
\sup _{\bar{\Omega}}|\epsilon u| \leq C
$$

Assuming $0 \in \Omega$, we consider $u-A|x|^{2}$. There is a large constant $A$ depending on $k, n$ and $\sup f$, such that

$$
F\left[D^{2} u\right]=f \leq F\left[D^{2}\left(A|x|^{2}\right)\right] .
$$

The maximum principle applying on (19) yields that $u-A|x|^{2}$ attains its minimum at some boundary point $x_{0}$. So

$$
0 \geq\left(u-A|x|^{2}\right)_{\nu}\left(x_{0}\right)=(-\epsilon u+\varphi-2 A x \cdot \nu)\left(x_{0}\right) .
$$

Similarly, since $u$ is a $k$ admissible solution, it is a subharmonic function. Then $u$ attains its maximum at some boundary point $y_{0}$. So

$$
0 \leq u_{\nu}\left(y_{0}\right)=(-\epsilon u+\varphi)\left(y_{0}\right) .
$$

It follows from (20) and (21) that

$$
\inf _{\partial \Omega} \varphi-4 A \operatorname{diam} \Omega \leq \epsilon u \leq \sup _{\partial \Omega} \varphi .
$$

Step 2. We prove the gradient estimate (16).

Without loss of generality, we assume $\int_{\Omega} u=0$ because otherwise we can use $v=u-f_{\Omega} u$ and $\tilde{\varphi}(x)=\varphi(x)-\epsilon f_{\Omega} u$ instead of $u$ and $\varphi$.

We Consider an auxiliary function

$$
P=\log |D w|^{2}+\alpha|x|^{2},
$$

where $w=u+(-\epsilon u+\varphi) d$ and $d=d(\cdot, \partial \Omega)$ is the distance function from $\partial \Omega$ defined in $\left\{x \in \mathbb{R}^{n} \mid d(x, \partial \Omega)<\mu\right\}$ for some small $\mu$ with smooth extension on $\bar{\Omega}$ so that $\|d\|_{C^{3}}$ is bounded, $\alpha$ is some positive constant to be determined later.

Suppose $P$ attains its maximum at an interior point $z_{0}$ of $\Omega$. At $z_{0}$, we have

$$
0=P_{i}=2 \frac{w_{l} w_{l i}}{|D w|^{2}}+2 \alpha x_{i}
$$

and

$$
0 \geq F^{i j} P_{i j}=\frac{2 F^{i j} w_{l j} w_{l i}}{|D w|^{2}}+\frac{2 F^{i j} w_{l} w_{l i j}}{|D w|^{2}}-\frac{4 F^{i j} w_{l} w_{l i} w_{p} w_{p j}}{|D w|^{4}}+2 \alpha \sum_{i} F^{i i}
$$


By the homogeneity of $F$ and taking first derivative of $F\left(D^{2} u\right)=f$, we have

$$
\begin{aligned}
F^{i j} u_{i j} & =k f, \\
F^{i j} u_{i j l} & =f_{l} .
\end{aligned}
$$

Taking derivatives to function $w$, we get

$$
\begin{gathered}
w_{i}=u_{i}+\left(-\epsilon u_{i}+\varphi_{i}\right) d+(-\epsilon u+\varphi) d_{i} \\
w_{i j}=u_{i j}+\left(-\epsilon u_{i j}+\varphi_{i j}\right) d+\left(-\epsilon u_{i}+\varphi_{i}\right) d_{j}+\left(-\epsilon u_{j}+\varphi_{j}\right) d_{i}+(-\epsilon u+\varphi) d_{i j}
\end{gathered}
$$

and

$$
\begin{aligned}
w_{i j l}= & u_{i j l}+\left(-\epsilon u_{i j l}+\varphi_{i j l}\right) d+\left(-\epsilon u_{i j}+\varphi_{i j}\right) d_{l} \\
& +\left(-\epsilon u_{i l}+\varphi_{i l}\right) d_{j}+\left(-\epsilon u_{i}+\varphi_{i}\right) d_{j l}+\left(-\epsilon u_{j l}+\varphi_{j l}\right) d_{i} \\
& +\left(-\epsilon u_{j}+\varphi_{j}\right) d_{i l}+\left(-\epsilon u_{l}+\varphi_{l}\right) d_{i j}+(-\epsilon u+\varphi) d_{i j l} .
\end{aligned}
$$

We choose the coordinate so that $|D w|=w_{1}$ and $\left(u_{i j}\right)_{2 \leq i, j \leq n}$ is diagonal at $z_{0}$. From (25), (26) and (29), we have

$$
\begin{aligned}
\frac{2 F^{i j} w_{l} w_{l i j}}{|D w|^{2}} \geq & \frac{2 w_{l}\left[(1-\epsilon) F^{i j} u_{i j l}-\epsilon F^{i j} u_{i j} d_{l}-2 \epsilon F^{i j} u_{i l} d_{j}\right]}{|D w|^{2}} \\
& -\frac{C \sum F^{i i}\left(1+w_{1}+\epsilon w_{1}^{2}\right)}{|D w|^{2}} \\
\geq & \frac{-C\left[\sum F^{i i}\left(1+w_{1}+\epsilon w_{1}^{2}\right)+w_{1}\right]-4 \epsilon F^{i j} u_{1 i} d_{j} w_{1}}{w_{1}^{2}},
\end{aligned}
$$

here $\epsilon$ is small, such that $\epsilon d<\frac{1}{2}$, constant $C$ depence on $\|\varphi\|_{C^{3}},\|f\|_{C^{1}}, n, k$, $\|\partial \Omega\|_{C^{3}}$ may be changed from line to line.

Using (23) and (28), we have

$$
\begin{aligned}
\left|u_{1 i}\right| & \leq C\left(\left|w_{1 i}\right|+\epsilon|D u|+1\right) \\
& \leq C(|D w|+1) .
\end{aligned}
$$

Using (31), we continue to compute (30) to get

$$
\frac{2 F^{i j} w_{l} w_{l i j}}{|D w|^{2}} \geq \frac{-C\left[\sum F^{i i}\left(1+w_{1}+\epsilon w_{1}^{2}\right)+w_{1}\right]}{w_{1}^{2}},
$$

On the other hand, we have by (23) again that

$$
\begin{aligned}
\frac{2 F^{i j} w_{l j} w_{l i}}{|D w|^{2}}-\frac{4 F^{i j} w_{l} w_{l i} w_{p} w_{p j}}{|D w|^{4}} & \geq-\frac{2 F^{i j} w_{1 i} w_{1 j}}{w_{1}^{2}} \\
& =-2 \alpha^{2} F^{i j} x_{i} x_{j} .
\end{aligned}
$$


If we choose $\alpha$ and $\epsilon$ such that $\frac{1}{\max _{\Omega}|x|^{2}} \geq \alpha \geq 2 C \epsilon$ and $|D w|$ sufficient large, we have from (32) and (33) that

$$
\begin{aligned}
F^{i j} P_{i j} & =\frac{2 F^{i j} w_{l j} w_{l i}}{|D w|^{2}}+\frac{2 F^{i j} w_{l} w_{l i j}}{|D w|^{2}}-\frac{4 F^{i j} w_{l} w_{l i} w_{p} w_{p j}}{|D w|^{4}}+2 \alpha \sum_{i} F^{i i} \\
& \geq \frac{-C\left[\sum F^{i i}\left(1+w_{1}+\epsilon w_{1}^{2}\right)+1\right]}{w_{1}^{2}}+2 \alpha \sum F^{i i}-2 \alpha^{2} F^{i j} x_{i} x_{j} \\
& >0 .
\end{aligned}
$$

This contradicts with (24). Thus $P$ can only attain its maximum at boundary points.

Suppose $P$ attains its maximum on a boundary point $\tilde{z}_{0}$. Choose a local orthonormal frame $\left\{\partial_{i}\right\}_{i=1}^{n}$ so that $\partial_{n}=\nu$. From the boundary condition, we have $w_{n}=0$ at $\tilde{z}_{0}$. By the maximal property of $P$ at $\tilde{z}_{0}$, we have

$$
0 \leq P_{\nu}=2 \frac{w_{l} w_{l \nu}}{|D w|^{2}}+2 \alpha x \cdot \nu=2 \frac{\sum_{\alpha=1}^{n-1} w_{\alpha} w_{\alpha n}}{|D w|^{2}}+2 \alpha x \cdot \nu .
$$
have

By taking the tangential derivative for the boundary condition along $\partial \Omega$, we

$$
u_{\alpha n}+\sum_{\beta=1}^{n-1} h_{\alpha \beta} u_{\beta}=-\epsilon u_{\alpha}+\varphi_{\alpha}, \quad \alpha=1, \cdots, n-1 .
$$

here $h_{\alpha \beta}$ is the second fundamental form of $\partial \Omega$. The equation (27) tells us ()

$$
(1-\epsilon d) u_{\alpha}-C \leq w_{\alpha} \leq(1-\epsilon d) u_{\alpha}+C,
$$

which also infer that

$$
\frac{1}{4} \sum_{\alpha=1}^{n-1} u_{\alpha}^{2}-C \leq|D w|^{2} \leq 4 \sum_{i=1}^{n-1} u_{\alpha}^{2}+C .
$$

Since $\Omega$ is uniformly convex, $h_{\alpha \beta} \geq c_{0} \delta_{\alpha \beta}$ for some $c_{0}>0$. Thus we deduce from (35) and (36) that

$$
\begin{aligned}
\sum_{\alpha=1}^{n-1} w_{\alpha} w_{\alpha n} & \leq-\frac{c_{0}}{4} \sum_{\alpha=1}^{n-1} u_{\alpha}^{2}-\epsilon|D w|^{2}-C|D w|-C \\
& \leq-\frac{c_{0}}{16}|D w|^{2}-\epsilon|D w|^{2}-C|D w|-C .
\end{aligned}
$$

By choosing $\epsilon \leq \frac{c_{0}}{32}, \alpha \leq \frac{c_{0}}{32 \max x \cdot \nu}$ and $|D w|$ sufficient large, we get

$$
P_{\nu} \leq-\frac{c_{0}}{16}+2 \alpha x \cdot \nu \leq 0 .
$$

This is a contradiction to (34). 
In conclusion we first choose $\alpha$ small and then $\epsilon$ small, we get two contradictions. That means, we cannot have $|D w|$ large. Hence we get the upper bound of $|D w|$ and in turn also $|D u|$.

Step 3. The a priori estimate (17) follows from the gradient estimate by the Poincare inequality. We complete the proof of Proposition 5 .

Now we readily prove Theorem 1

Proof. Let $u_{\epsilon}$ be a solution of (7) for any $\epsilon>0$. Because $|\nabla(-\epsilon u)| \rightarrow 0$ and the Schauder estimate, there is a constant $\lambda$ and a function $\bar{u} \in C^{2}(\bar{\Omega})$, such that

$$
-\epsilon u \rightarrow \lambda, \text { and } \quad u_{\epsilon}-f_{\Omega} u_{\epsilon} \rightarrow \bar{u} \text { uniformly in } C^{2} \text { as } \epsilon \rightarrow 0 .
$$

It follows that $\bar{u}$ solves the following classical Neumann problem

$$
\begin{cases}\sigma_{k}\left(D^{2} u\right)=f(x) & \text { in } \Omega \\ u_{\nu}=\lambda+\varphi(x) & \text { on } \partial \Omega\end{cases}
$$

Next we prove the uniqueness. Suppose problem (37) has two pairs of solutions $(\lambda, u)$ and $(\mu, v)$. Let $a^{i j}=\int_{0}^{1} F^{i j}\left[(1-t) D^{2} v+t D^{2} u\right] d t$, and $u-v$ satisfies

$$
\left\{\begin{array}{l}
a^{i j}(u-v)_{i j}=0, \\
(u-v)_{\nu}=\lambda-\mu .
\end{array}\right.
$$

It follows that $u-v$ attains its maximum and its minimum both at some boundary points. It shows that $\lambda=\mu$. Finally, the Hopf lemma in [6, Theorem 3.6] yields $u-v=c$.

\section{Alexandrov-Fenchel Inequalities}

In this chapter, we use the solution of the classical Neumann problems to give a new proof of some Alexandrov-Fenchel inequalities. We need the following result due to Reilly [16].

Proposition 7. Let $\Omega$ be a smooth, bounded convex domain in $\mathbb{R}^{n}$. Let $u \in$ $C^{2}(\bar{\Omega})$ such that $\lambda\left(D^{2} u\right) \in \Gamma_{k}^{+}$and $u_{\nu}=c$ on $\partial \Omega$, where $c$ is a positive constant, then the following inequality holds:

$$
(k+1) \int_{\Omega} \sigma_{k+1}\left(D^{2} u\right) \geq \int_{\partial \Omega} \sigma_{k}(h) c^{k+1} .
$$

Proof. For completeness, we give a proof here.

In the following we choose an orthonormal coordinate $\left\{\partial_{i}\right\}_{i=1}^{n}$ such that $\partial_{n}=\nu$ on the boundary. We denote by $D_{i j}^{2} u$ the Hessian with respect to the 
ambient (Euclidean) metric, $u_{\alpha \beta}=\nabla_{\alpha \beta}^{2} u$ the Hessian of $u$ with respect to the induced metric on $\partial \Omega$ and $u_{n \alpha}=\nabla_{\alpha}\left(u_{n}\right)$ on $\partial \Omega$. We will sum the repeated indices as before and also the convention that the Latin indices run through 1 to $n$ while the Greek indices run through 1 to $n-1$.

It follows from the Gauss-Weingarten formula that

$$
\begin{aligned}
& D_{\alpha \beta}^{2} u=u_{\alpha \beta}+h_{\alpha \beta} u_{n}, \\
& D_{\alpha n}^{2} u=u_{n \alpha}-h_{\alpha \beta} u_{\beta} .
\end{aligned}
$$

Set $A$ and $B$ to be the following two matrices:

$$
\begin{gathered}
A:=\left(\begin{array}{cccc}
\cdots & \cdots & \cdots & \vdots \\
\cdots & u_{\alpha \beta} & \cdots & u_{n \alpha} \\
\cdots & \cdots & \cdots & \vdots \\
\cdots & u_{n \alpha} & \cdots & u_{n n}
\end{array}\right), \\
B:=\left(\begin{array}{cccc}
\cdots & \cdots & \cdots & \vdots \\
\cdots & h_{\alpha \beta} u_{n} & \cdots & -h_{\alpha \gamma} u_{\gamma} \\
\cdots & \ldots & \cdots & \vdots \\
\cdots & -h_{\beta \gamma} u_{\gamma} & \cdots & 0
\end{array}\right) .
\end{gathered}
$$

Then on $\partial \Omega, D^{2} u$ is decomposed to be

$$
D^{2} u=A+B
$$

By using (12) and (15) we see

$(k+1) \int_{\Omega} \sigma_{k+1}\left(D^{2} u\right)=\int_{\partial \Omega}\left[T_{k}\right]_{i n}\left(D^{2} u\right) D_{i j}^{2} u=\int_{\partial \Omega}\left[T_{k}\right]_{i n}\left(D^{2} u\right) u_{i}=I+I I$,

where

$$
I:=\int_{\partial \Omega}\left[T_{k}\right]_{n n}\left(D^{2} u\right) u_{n}, \quad \text { and } \quad I I:=\int_{\partial \Omega}\left[T_{k}\right]_{\alpha n}\left(D^{2} u\right) u_{\alpha} .
$$

Note that

$$
\left[T_{k}\right]_{n n}\left(D^{2} u\right)=\frac{1}{k !} \sum_{\substack{\alpha_{1}, \cdots \alpha_{k}, \beta_{1}, \cdots, \beta_{k}}} \delta_{\alpha_{1} \cdots \alpha_{k}}^{\beta_{1} \cdots \beta_{k}} D_{\alpha_{1} \beta_{1}}^{2} u \cdots D_{\alpha_{k} \beta_{k}}^{2} u=\sigma_{k}\left(\left.D^{2} u\right|_{\partial \Omega}\right)
$$

and

$$
\begin{aligned}
{\left[T_{k}\right]_{\alpha n}\left(D^{2} u\right) } & =-\frac{1}{(k-1) !} \sum_{\substack{\alpha_{1}, \cdots \alpha_{k-1}, \beta_{1}, \cdots, \beta_{k-1} \neq \alpha}} \delta_{\alpha \alpha_{2} \cdots \alpha_{k-1}}^{\beta_{1} \beta_{2} \cdots \beta_{k-1}} D_{\alpha_{2} \beta_{2}}^{2} u \cdots D_{\alpha_{k-1} \beta_{k-1}}^{2} u D_{\beta_{1} n}^{2} u \\
& =\left[T_{k-1}\right]_{\alpha \beta_{1}}\left(\left.D^{2} u\right|_{\partial \Omega}\right) D_{\beta_{1} n}^{2} u
\end{aligned}
$$


Using the assumption $u_{n}=c$, we know $\nabla_{\beta_{1}} u_{n}=0$ and hence $D_{\beta_{1} n}^{2} u=$ $-h_{\beta_{1} \alpha_{1}} u_{\alpha_{1}}$. By using the decomposition $D^{2} u=A+B$ and (14), we have

$$
\begin{aligned}
I I & =\int_{\partial \Omega}\left[T_{k-1}\right]_{\alpha \beta_{1}}\left(\left.D^{2} u\right|_{\partial \Omega}\right) h_{\alpha_{1} \beta_{1}} u_{\alpha_{1}} u_{\alpha} \\
& =\int_{\partial \Omega} \sum_{l=0}^{k-1} C_{k-1}^{l}\left[T_{k-1}\right]_{\alpha \beta_{1}}(\overbrace{\nabla^{2} u, \cdots, \nabla^{2} u}^{l}, h, \cdots, h) u_{n}^{k-1-l} h_{\alpha_{1} \beta_{1}} u_{\alpha_{1}} u_{\alpha} .
\end{aligned}
$$

Similarly, we have

$$
\begin{aligned}
I & =\int_{\partial \Omega} \sum_{l=1}^{k} C_{k}^{l} \sigma_{k}(\overbrace{\nabla^{2} u, \cdots, \nabla^{2} u}^{l}, h, \cdots, h) u_{n}^{k-l+1}+\int_{\partial \Omega} \sigma_{k}(h) u_{n}^{k+1} \\
& =: \quad I_{1}+I_{2} .
\end{aligned}
$$

The term $I_{2}$ is what we want in final. We compute $I_{1}$ further. By intergration by parts and using again $u_{n}=c$, we get

$$
\begin{aligned}
I_{1} & =\int_{\partial \Omega} \sum_{l=1}^{k} C_{k}^{l} \sigma_{k} \overbrace{\nabla^{2} u, \cdots, \nabla^{2} u}^{l}, h, \cdots, h) u_{n}^{k-l+1} \\
& =-\int_{\partial \Omega} \sum_{l=2}^{k} C_{k}^{l} \frac{l-1}{k !} \delta_{\beta_{1} \cdots \beta_{k}}^{\alpha_{1} \cdots \alpha_{k}} u_{\alpha_{1}} \cdots u_{\alpha_{l} \beta_{l} \beta_{1}} h_{\alpha_{l+1} \beta_{l+1}} \cdots h_{\alpha_{k} \beta_{k}} u_{n}^{k-l+1}
\end{aligned}
$$

where in the second equality we also used the fact $h_{\alpha_{k} \beta_{k} \beta_{1}}=h_{\alpha_{k} \beta_{1} \beta_{k}}$, which is the Codazzi property of the second fundamental form $h_{\alpha \beta}$.

The Ricci identity tells that

$$
u_{\alpha_{l} \beta_{l} \beta_{1}}-u_{\alpha_{l} \beta_{1} \beta_{l}}=u_{\gamma} R_{\gamma \alpha_{l} \beta_{l} \beta_{1}}=u_{\gamma} h_{\gamma \beta_{l}} h_{\alpha_{l} \beta_{1}}-u_{\gamma} h_{\gamma \beta_{1}} h_{\alpha_{l} \beta_{l}} .
$$

Replacing the above into (41), we have

$$
\begin{aligned}
I_{1} & =\int_{\partial \Omega} \sum_{l=2}^{k} C_{k}^{l} \frac{l-1}{k !} \delta_{\beta_{1} \cdots \beta_{k}}^{\alpha_{1} \cdots \alpha_{k}} u_{\alpha_{1}} u_{\alpha_{2} \beta_{2}} \cdots u_{\alpha_{l-1} \beta_{l-1}} u_{\gamma} h_{\gamma \beta_{1}} h_{\alpha_{l} \beta_{l}} h_{\alpha_{l+1} \beta_{l+1}} \cdots h_{\alpha_{k} \beta_{k}} u_{n}^{k-l+1}, \\
& =\int_{\partial \Omega} \sum_{l=2}^{k} C_{k}^{l} \frac{l-1}{k}\left[T_{k-1}\right]_{\alpha_{1} \beta_{1}}(\overbrace{\nabla^{2} u, \cdots, \nabla^{2} u}^{l-2}, h, \cdots, h) u_{\alpha_{1}} u_{\gamma} h_{\gamma \beta_{1}} u_{n}^{k-l+1} \\
& =\int_{\partial \Omega} \sum_{l=0}^{k-1} C_{k}^{l+2} \frac{l+1}{k}\left[T_{k-1}\right]_{\alpha_{1} \beta_{1}}(\overbrace{\nabla^{2} u, \cdots, \nabla^{2} u}^{l+1}, h, \cdots, h) u_{\alpha_{1}} u_{\gamma} h_{\gamma \beta_{1}} u_{n}^{k-l-1} .
\end{aligned}
$$

We sum $I I$ and $I_{1}$ to be $I I I$. 


$$
\begin{aligned}
I I I & :=I_{1}+I I \\
& =\int_{\partial \Omega} \sum_{l=0}^{k-1} C_{k+1}^{l+2} \frac{l+1}{k}\left[T_{k-1}\right]_{\alpha_{1} \beta_{1}}(\overbrace{\nabla^{2} u, \cdots, \nabla^{2} u}^{l}, h, \cdots, h) u_{\alpha_{1}} u_{\gamma} h_{\gamma \beta_{1}} u_{n}^{k-l-1} .
\end{aligned}
$$

In order to see the sign of $I I I$, we use $\nabla_{\alpha \beta}^{2} u=D_{\alpha \beta}^{2} u-h_{\alpha \beta} u_{n}$ in (39) to get

$$
\begin{aligned}
I I I & =\int_{\partial \Omega} \sum_{l=0}^{k-1} \sum_{i=0}^{l}(-1)^{l-i} C_{k+1}^{l+2} C_{l}^{i} \frac{l+1}{k}\left[T_{k-1}\right]_{\alpha_{1} \beta_{1}} \overbrace{D^{2} u, \cdots, D^{2} u}^{i}, h, \cdots, h) u_{\alpha_{1}} u_{\gamma} h_{\gamma \beta_{1}} u_{n}^{k-i-1} \\
& =\int_{\partial \Omega} \sum_{i=0}^{k-1} \sum_{l=i}^{k-1}(-1)^{l-i} C_{k+1}^{l+2} C_{l}^{i} \frac{l+1}{k}\left[T_{k-1}\right]_{\alpha_{1} \beta_{1}} \overbrace{D^{2} u, \cdots, D^{2} u}^{i}, h, \cdots, h) u_{\alpha_{1}} u_{\gamma} h_{\gamma \beta_{1}} u_{n}^{k-i-1} .
\end{aligned}
$$

If we can prove that $I I I \geq 0$, we are done. We see first that since $\lambda\left(D^{2} u\right) \in$ $\Gamma_{k}^{+}$and $h_{\alpha \beta}$ is nonnegative,

$$
\left[T_{k-1}\right]_{\alpha_{1} \beta_{1}}(\overbrace{D^{2} u, \cdots, D^{2} u}^{i}, h, \cdots, h) h_{\gamma \beta_{1}} u_{\alpha_{1}} u_{\gamma} \geq 0
$$

by the Garding inequality (See [5]).

Hence to prove $I I I \geq 0$, we need to only to prove

$$
E:=\sum_{l=i}^{k-1}(-1)^{l-i} C_{k+1}^{l+2} C_{l}^{i} \frac{l+1}{k} \geq 0 .
$$

Following a trick in [14], we deal this term as follows:

$$
\begin{aligned}
E & =(k+1) C_{k-1}^{i} \sum_{l=i}^{k-1} \frac{1}{l+2} C_{k-1-i}^{l-i}(-1)^{l-i} \\
& =(k+1) C_{k-1}^{i} \sum_{p=0}^{k-1-i} \frac{1}{p+i+2} C_{k-1-i}^{p}(-1)^{p}
\end{aligned}
$$

Note that the folowing elementary equality holds:

$$
\begin{aligned}
\int_{0}^{1} t^{i+1}(1-t)^{k-i-1} d t & =\int_{0}^{1} t^{1+i} \sum_{p=0}^{k-i-1} C_{k-i-1}^{p}(-1)^{p} t^{p} d t \\
& =\left.\sum_{p=0}^{k-i-1} C_{k-i-1}^{p}(-1)^{p} \frac{t^{p+2+i}}{p+2+i}\right|_{t=0} ^{t=1}
\end{aligned}
$$


Hence

$$
E=(k+1) C_{k-1}^{i} \int_{0}^{1} t^{1+i}(1-t)^{k-1-i} d t=\frac{i+1}{k} .
$$

Finally, we conclude

$$
\begin{aligned}
& \int_{\partial \Omega}\left[T_{k}\right]_{i j}\left(D^{2} u\right) u_{i} \nu_{j} d \mu \\
= & \int_{\partial \Omega} \sigma_{k}(h) u_{n}^{k+1}+\sum_{i=0}^{k-1} \frac{i+1}{k}\left[T_{k-1}\right]_{\alpha_{1} \beta_{1}} \overbrace{D^{2} u, \cdots, D^{2} u}^{i}, h, \cdots, h) h_{\gamma \beta_{1}} u_{\alpha_{1}} u_{\gamma} u_{n}^{k-i-1} \\
\geq & \int_{\partial \Omega} \sigma_{k}(h) u_{n}^{k+1} .
\end{aligned}
$$

The proof of Proposition 7 is completed.

Proof of Theorem 2, From Theorem[1] there is a $k$ admissible solution $u$ and an unique constant $c$ satisfying

$$
\begin{cases}\sigma_{k}\left(D^{2} u\right)=C_{n}^{k} & \text { in } \Omega, \\ u_{\nu}=c & \text { on } \partial \Omega .\end{cases}
$$

On one hand, since $\lambda\left(D^{2} u\right) \in \Gamma_{k}^{+}$, the Newton-Maclaurin inequality (11) that

$$
\int_{\Omega}(k+1) \sigma_{k+1}\left(D^{2} u\right) \leq \int_{\Omega}(k+1) C_{n}^{k+1}\left(\frac{\sigma_{k}\left(D^{2} u\right)}{C_{n}^{k}}\right)^{\frac{k+1}{k}} .
$$

Using Proposition 7 (46) and equation (45), we have

$$
c^{k+1} \int_{\partial \Omega} \sigma_{k}(h) \leq(k+1) C_{n}^{k+1} \operatorname{Vol}(\Omega) .
$$

On the other hand the Newton-MacLaurin inequality (11) also that

$$
\int_{\Omega}\left(\frac{\sigma_{k}\left(D^{2} u\right)}{C_{n}^{k}}\right)^{\frac{1}{k}} \leq \int_{\Omega} \frac{\sigma_{1}\left(D^{2} u\right)}{n}=\int_{\partial \Omega} \frac{u_{\nu}}{n}=c \frac{\operatorname{Area}(\partial \Omega)}{n} .
$$

Combine (47) and (48), we conclude

$$
\operatorname{Vol}(\Omega)^{k} \int_{\partial \Omega} \sigma_{k}(h) \leq \frac{C_{n-1}^{k}}{n^{k}} \operatorname{Area}(\partial \Omega)^{k+1} .
$$

When equality attains in the above inequality, from the proof in Proposition 7. we know that the tangential derivative of $u$ vanishes. Also we see from (47) that $\left(\frac{\sigma_{k}\left(D^{2} u\right)}{C_{n}^{k}}\right)^{\frac{1}{k}}=\frac{\sigma_{1}\left(D^{2} u\right)}{n}$. So $u$ satisfies the overdetermined problem

$$
\left\{\begin{array}{lll}
\Delta u=n & \text { in } & \Omega \\
u_{\nu}=c & \text { on } & \partial \Omega \\
u=b & \text { on } & \partial \Omega
\end{array}\right.
$$


where $b$ is a constant.

By using the result of Serrin [19, we have that (10) becomes equality if and only $\Omega$ is a ball. The proof is completed.

Acknowledgement. The first author would like to express gratitude to Prof. Xinan Ma for his advice and constant encouragement. Both authors would like to thank Prof. Pengfei Guan for valuable discussions and support.

\section{References}

[1] Alexander Alexandroff. Zur theorie der gemischten volumina von konvexen korpern. ii. neue ungleichungen zwischen den gemischten volumina und ihre anwendungen. Matematicheskii Sbornik, 44(6):1205-1238, 1937.

[2] Xavier Cabre. Elliptic PDE's in probability and geometry: symmetry and regularity of solutions. Discrete and Continuous Dynamical Systems. Series A 20(3): 425-457, 2008.

[3] Sun-Yung A Chang and Yi Wang. Inequalities for quermassintegrals on k-convex domains. Advance in Mathematics, 248: 335-377, 2013.

[4] Sun-Yung A Chang and Yi Wang. Some higher order isoperimetric inequalities via the method of optimal transport. International Mathematics Research Notices, page rnt182, 2013.

[5] Lars Garding. An inequality for hyperbolic polynomials. J. Math. Mech. 8: 957-965, 1959.

[6] David Gilbarg and Neil S Trudinger. Elliptic partial differential equations of second order. Springer-Verlag, Berlin Heidelberg NewYork Tokyo, 1983.

[7] M Gromov. Isometric immersions of Riemannian manifolds. Astérisque Numero Hors Serie, 129-133, 1985.

[8] Pengfei Guan and Junfang Li. The quermassintegral inequalities for kconvex starshaped domains. Advances in Mathematics, 221(5):1725-1732, 2009 .

[9] Pengfei Guan Topics in Geometric Fully Nonlinear Equations, available at "http://www.math.mcgill.ca/guan/notes.html"

[10] P-L Lions, NS Trudinger, and JIE Urbas. The neumann problem for equations of monge-ampére type. Communications on Pure and Applied Mathematics, 39(4):539-563, 1986.

[11] Xinan Ma and Guohuan Qiu. The neumann problem for hessian equations. arXiv preprint arXiv:1508.00196, 2015. 
[12] Xinan Ma, Guohuan Qiu, and Jinju Xu. Gradient estimates on hessian equations for neumann problem. SCIENTIA SINICA Mathematica, 2016.

[13] Giacomo Nardi. Schauder estimate for solutions of Poisson's equation with Neumann boundary condition. L'Enseignement Mathematique, 60 (3-4): 421-435, 2014.

[14] Guohuan Qiu. A family of higher-order isoperimetric inequalities. Communications in Contemporary Mathematics, 17(03):1450015, 2015.

[15] Robert C Reilly. On the Hessian of a function and the curvatures of its graph. Michigan Math. J. 20: 373-383, 1973.

[16] Robert C Reilly. Applications of the Hessian operator in a Riemannian manifold. Indiana Univ. Math. J, 26(3):459-472, 1977.

[17] Robert C Reilly. Geometric applications of the solvability of Neumann problems on a Riemannian manifold. Arch. Rational Mech. Anal. 75(1): 23-29, 1980.

[18] Rolf Schneider. Convex bodies: the Brunn-Minkowski theory. Number 151. Cambridge University Press, 2013.

[19] James Serrin. A symmetry problem in potential theory. Archive for Rational Mechanics and Analysis, 43(4):304-318, 1971.

[20] Neil S Trudinger. On degenerate fully nonlinear elliptic equations in balls. Bulletin of the Australian Mathematical Society, 35(02):299-307, 1987.

[21] Neil S Trudinger. Isoperimetric inequalities for quermassintegrals. In $A n$ nales de l'IHP Analyse non lineaire, volume 11, pages 411-425, 1994.

[22] Yu Wang and Xiangwen Zhang. An Alexandroff-Bakelman-Pucci estimate on riemannian manifolds. Advances in Mathematics, 232(1):499-512, 2013.

[23] Chao Xia. A Minkowski type inequality in space forms. Calc. Var. PDE, to appear, 2016.

[24] Chao Xia and Xiangwen Zhang. ABP estimate and geometric inequalities. Communications in Analysis and Geometry, to appear, 2016. 